\title{
EXPERIMENTAL REPRODUCTION OF THE SPECIFIC HISTOPATHOLOGY OF INFLUENZA *
}

\author{
GEORGE BAEHR, M.D., AXd LEO LOEWE, M.D. \\ NEW YORK
}

It was realized rather early in the recent pandemic of influenza that by the time patients died secondary infections with various pyogenic organisms had usually taken place, which more or less completely obliterated evidences of the original disease. From numerous bacteriologic studies, it was evident that the bacteria recovered from the lungs after death had secondarily invaded the damaged organs from the upper respiratory tract. Traditional belief made many observers reluctant to assign a similar rôle to the $B$. influen a a of Pfeiffer. But even this organism was finally relegated to a secondary rôle by work such as that of MacCallum, ${ }^{1}$ which demonstrated that although it might be present in a large percentage of the lungs of persons dying of the disease in one part of the country, the bacillus of Pfeiffer was correspondingly rare in the lungs in other parts of the country where, at the time, it happened to be a less frequent resident of the upper respiratory tract.

Such advances in our knowledge of the bacteriology of the disease immediately concentrated the efforts of pathologists on an attempt to differentiate the secondary purulent from the primary fundamental lesions of epidemic influenza. The best work in this country (Klotz; ${ }^{2}$ Winternitz, Wason and MacNamara ${ }^{3}$ ) kept this aim constantly in view, as did the best of the European observers (Oberndorter." Dietrich, ${ }^{5}$ Glaus and Fritzche, ${ }^{6}$ Marchand, ${ }^{7}$ Soerensen, ${ }^{8}$ Koopman ${ }^{9}$ ). Some of these authors (Klotz, Oberndorfer, Glaus and Fritzsche, Koopman) had the unusual opportunity to study fulminating cases of the disease in persons who had died within forty-eight hours after the onset, before the secondary infection had sufficiently invaded the damaged lung to obscure the primary pathological picture.

* From the Pathological Department of the Mount Sinai Hospital.

* Work done during the tenure of a George Blumenthal, Jr. fellowship in pathology.

1. MacCallum: J. A. M. A. 72:720 (March 8) 1919.

2. Klotz: Studies on Epidemic Influenza. Published from University of Pittsburgh School of Medicine, 1919, p. 207.

3. Winternitz, Wason and MacNamara: The Pathology of Influenza, Yale University Press, 1920.

4. Oberndorfer: München. med. Wchnschr. 65:30, 1918.

5. Dietrich: München. med. Wchnschr. 65:34, 1918.

6. Glaus and Fritzsche: Cor.-B1. f. schweiz. Aerzte 48:1121, 1918; ibid. $49: 72,1919$.

7. Marchand: München. med. Wchnschr. 66:5, 1919.

8. Soerensen: Ztsclir. f. Hygiene u. Infectionskr. 91:204, 1921.

9. Koopman: Virchows Arch. f. path. Anat. 228:319. 1920. 
Specificity of the Histopathology of Early Human Influenza.Summarizing the work of these authors, one is impressed with the unanimity of their opinion that the pathologic process in early human influenza represents a specific tissue reaction, not to be seen in the lungs in any other type of infection. In fact, Winternitz and his collaborators state in their monograph: "The histology of this disease is almost as specific as that of any biologic reaction."

The essential features of the pathologic lesions in early human influenza are (1) congestion, edema and often minute hemorrhages in the mucous membrane of the trachea and larger bronchi; (2) patchy distribution of the pulmonary lesions; (3) diffuseness of the process, which often extends through several lobes or even parts of all lobes; (4) profuse serosanguinous exudate which drips from the moist cut surface of involved parenchyma; (5) hemorrhagic character of the lesion, the hemorrhagic exudate being seen microscopically to infiltrate the interstitial tissue and fill patches of alveoli; (6) aplastic character of the process, the inflammatory exudate in alveoli and interalveolar interstitial tissue being composed almost entirely of red cells and serum with an occasional endothelial cell but containing astonishingly few polymorphonuclear or other leukocytes. In addition, Klotz directed special attention to the tendency to emphysematous overdistension of alveolar air spaces in the involved lungs; and Winternitz also emphasized the tendency to necrosis of the epithelium lining the trachea and bronchi and to hyalin necrosis of pulmonary tissue, especially of interalveolar septa in the damaged areas. One of us had considerable experience with the study of this unusual type of pulmonary inflammatory process, while serving during the pandemic as director of laboratories for the group of U. S. Army Base Hospitals at Beau Désért Hospital Center, France.

Experimental Reproduction of Influenza.-Just as advances in bacteriology during the first year of the pandemic (1918) were in large part responsible for a more accurate conception of the pathologic phenomena involved in influenza, so, in similar manner, the more recent bacteriologic investigations of Olitsky and Gates ${ }^{10}$ and of Loewe and Zeman ${ }^{11}$ have afforded renewed opportunities for a study of the essential features of this pathologic process. Independently of one another and simultaneously, these two groups of investigators succeeded in cultivating a filtrable virus from the nasopharyngeal washings made early in the course of the influenza, which is capable of producing lesions in the lungs of rabbits, grossly and microscopically identical with those found in human beings suffering from the disease.

10. Olitsky and Gates: J. Exper. M. 33:125, 1921.

11. Loewe and Zeman: J. A. M. A., 76:986 (April 9) 1921. 
We have, therefore, undertaken to study more minutely the pathologic process induced experimental in these animals by the intra tracheal inoculation of cultures of these punctiform, filtrable bodies and of influenza virus (filtered human nasopharygeal washings or lungs from inoculated animals). In this manner we have been able to observe the lesions at the very earliest stages of their development, an

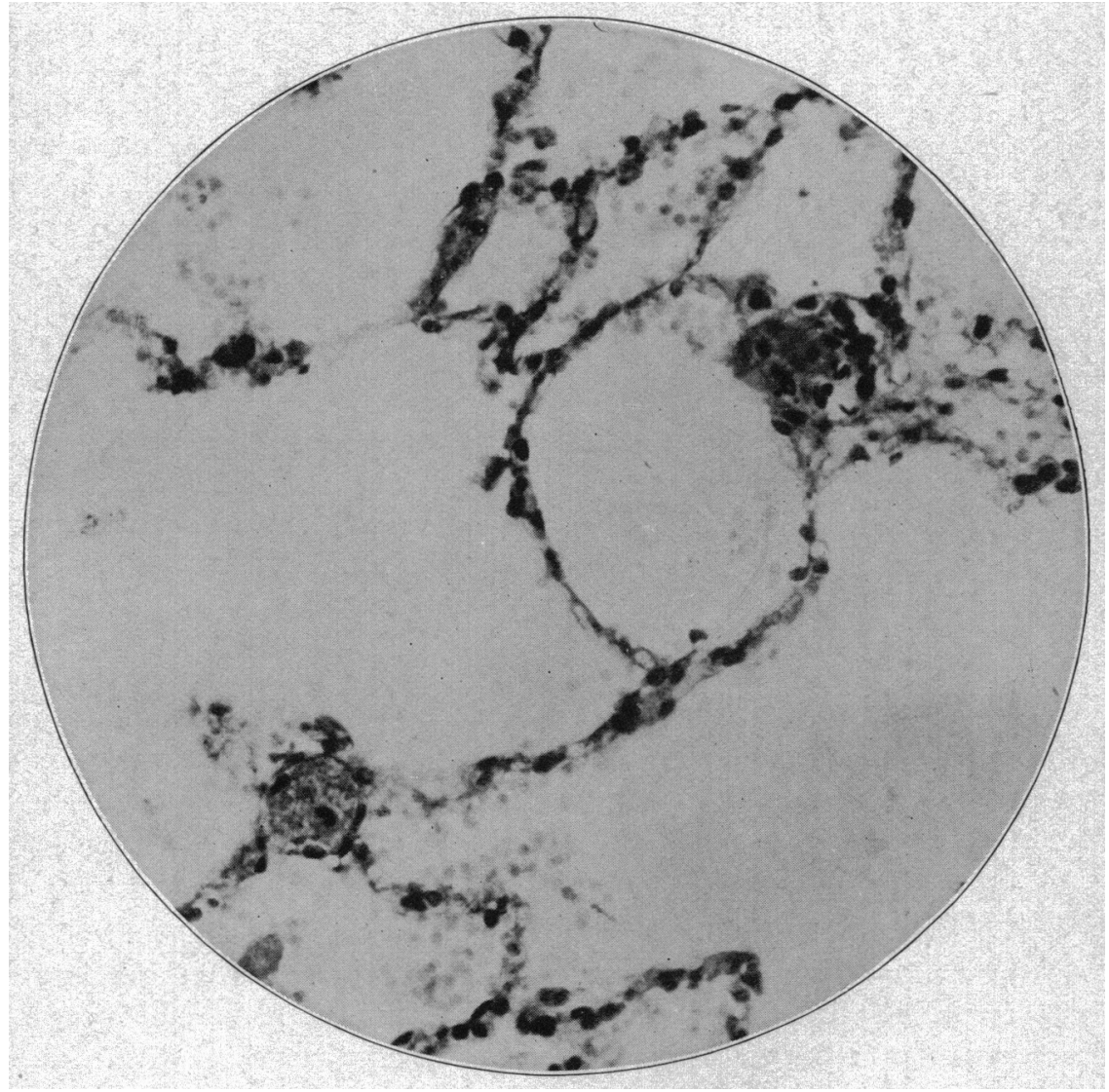

Fig. 1.-Section from portion of lung showing minimal amount of parenchymal damage. Shows (1) emphysematous overdistension of alveoli, (2) congestion, (3) beginning extravasation of red blood cells into the alveoli, (4) two capillaries cut in cross section which are widely dilated and are occluded by blood platelet thrombi. (X400.)

opportunity obviously impossible in human beings. And this opportunity has enabled us, we believe, to throw more light upon the pathogenesis of the disease.

Materials employed.-Four series of rabbits were inoculated intratracheally with the following materials:

(1) Human nasopharyngeal washings: In the first series, animals were inoculated with nasopharyngeal washings from early cases of 
influenza. These washings were obtained in the manner described elsewhere, ${ }^{11}$ within twenty-four hours after the onset of the first symptoms of illness, and they were then carefully filtered under strict sterile precalutions through Berkefeld filters size $\mathrm{N}$ or Mandler filters of corresponding density. The filters were always tested with $B$. prodigiosus. The filtered nasopharyngeal washings were then cultured aerobically and anaerobically in ordinary laboratory mediums and found to be free of contaminations. Inoculations were also made on the so-called chocolate agar medium in order to eliminate the possible presence of $B$. inflenzae of Pfeiffer or other hemoglobinophilic organisms. From 1 to 3 c.c. of the clear filtrate were used for the intratracheal inoculations. the material being introduced in the manner subsequently to be described.

(2) Filtrates of glycerolated lungs of inoculated animals: In a second series, the injected material consisted of the lungs of animals in series one and three, which had been glycerolated for a variable period (from one week to fourteen months) and then filtered through Berkefeld or Mandler filters. The lungs were removed in sterile fashion from the animals within from thirty-six to forty-eight hours after inoculation and were not further utilized unless found to be free of all secondary invading organismis. Glycerolation was often employed as an added precaution as it did not seem to affect the virulence of the material appreciably.

(3) Cultures of the punctiform bodies. In the third series, the rabbits were inoculated intratracheally with cultures of the minute punctiform filtrable bodies isolated by Loewe and Zeman. Most of the cultures were obtained from the filtered nasopharyngeal washing: of early cases of influenza, persons in the first twenty-four or thirtysix hours of their illness. Other cultures were derived from the lungs; of animals which had originally been inoculated intratracheally with the filtered nasopharyngeal washings of early human cases of influenza. And a third group of cultures employed was recovered from the lungs of animals in which the experimental disease had been produced by the inoculation of cultures isolated from stich nasopharyngeal washings.

One interesting feature of this third series of experiments is that the cultures of this filtrable virus, after being kept at incubator temperature for fourteen months, were not only found to be still viable, but also retained their unique pathogenic properties. Similarly, the lungs of animals in which the disease had been produced by intratracheal inoculation of human nasopharyngeal washings could be preserved in 50 per cent. glycerol, and after fourteen months the glycerolated material was viable and also retained an almost undiminished infectivity.

(4) Control experiments: The fourth series of experiments served as controls to series one, two and three. The inoculated materials in this series consisted of the following: 
(a) Culture medium: ascitic fluid containing lidney tissue and layered with petrolatum, which had not been inoculated but had been incubated at 37 C. simultaneous'y with the inoculated cultures for varying periods of time up to fourteen months.

(b) Berkefeld filtrates of glycerolated lungs of rabbits succumbing to intercurrent laboratory infections or to the intratracheal inoculation of various pyogenic organisms.

(c) Filtrates of nasopharyngeal washings obtained in the customary manner from patients suffering with diseases other than influenza. Surgical cases were preferred to rule out possible contact infection.

(d) Cultures of Staphylococcus aurcus, Streptococcus hemolyticus, Streptococcus anhemolyticus, pneumococcus and Bacillus bipolaris (distemper).

(e) Cultures of $B$. influenzae of Pfeiffer which were derived from various sources such as (1) nasopharynx of normal individuals; (2) cerebrospinal fluid of influenzal meningitis; (3) nasopharynx, sputum and lungs of persons suffering from influenza during the pandemic. ${ }^{12}$

Tochnic of Inoculation.- The rabbit, having been lightly anesthetized with ethyl chlorid, ${ }^{13}$ from 1 to $3 \mathrm{cc}$. of culture or other material was inoculated intratracheally. In a few experiments this was accomplished by passing a fine rubber catheter into the trachea in the manner of the Meltzer-Auer method of intratracheal insufflation. It was found to be more satisfactory to inject the material directly through the tracheal wall by means of a fine needle and syringe, after previous removal of the hair with barium sulphide and sterilization of the skin with tincture of iodin. ${ }^{14}$ The animals immediately recovered from the very transient anesthesia and presented no evidence of any distress.

Symptoms of Illness.-Often the first evidences of illness made their appearance within a few hours after the inoculation. Within twenty-four hours, the animals appeared to be severely ill, their movements were less active, breathing was more rapid, and the conjunctivae were injected. Over both sides of the chest. the stethoscope elicited numerous moist räles and, in some animals a distinct expiratory, asthma-like wheeze.

Leukopenia.-Simultaneous with the development of these symptoms, there appeared a pronounced leukopenia, the number of leukocytes in the blood at times decreasing from 50 to 60 per cent. within

12. For many of the strains we are indebted to Dr. William H. Park of the Research Laboratory of the New York City Board of Health.

13. Ethyl chlorid was employed owing to the ease and rapidity with which a light anesthesia could be induced. It was proven that this volatile drug as used was incapable of producing any structural changes.

14. Direct intratracheal inoculation was performed with 18-gage needles connected to 5 c.c. Luer syringes. Entrance into the trachea was indicated by the easy withdrawal of air upon elevating the plunger of the syringe. 
forty-eight hours. The leukopenia was present in every instance after the inoculation of either active influenza virus (filtered lungs or nasopharyngeal washings), or cultures obtained from the virus. It was never encountered in animals inoculated with pyogenic organisms or with $B$. influenzae of Pfeiffer; on the contrary, even the latter regularly induced a definite hyperleukocytosis.

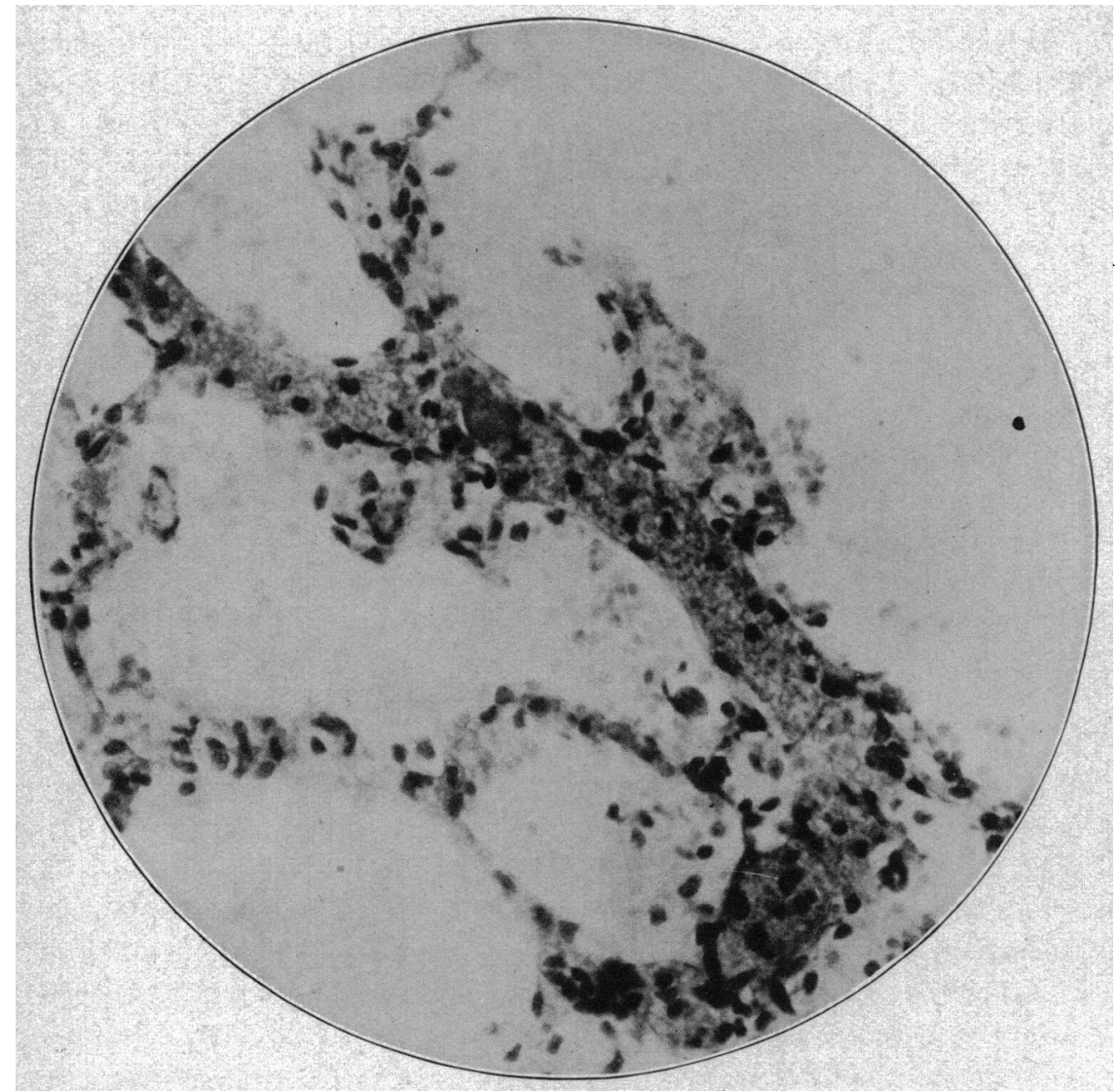

Fig. 2.-Section showing an occluded arteriole and its tributary capillaries cut longitudinally. The outlines of the blood platelets comprising the thrombus can be made out, even with this magnification. Although the thrombus contains a few polymorphonuclear and mononuclear leukocytes, the cellular forms of inflammatory exudate are conspicuously absent in the alveolar air spaces and interalveolar tissue. In this microscopic field the alveolar spaces contain serum and red blood cells, whereas in other portions of the same section the interstitial tissue is the more extensively infiltrated with the aplastic serosanguineous exudate. $(\times 400$.

Secondary Incasion with Pyogenic Organisms.-At the very beginning of the work, it was immediately observed that animals which were permitted to live longer than from forty-eight to seventy-two hours 
after the onset of the disease usually developed various types of secondary infection within their damaged lung parenchyma. Exactly as in human influenza, the primary process then became obscured by complicating lesions such as pneumonic consolidations, abscesses, empyema, etc., from which various pyogenic organisms could be isolated. Simultaneous with the onset of this secondary bacterial invasion, the leukopenia rapidly gave way to a hyperleukocytosis.

It was therefore necessary to confine the study to the lungs of rabbits which were sacrificed ${ }^{15}$ within from twenty-four to forty-eight hours after the inoculation. The following observations therefore represent the earliest lesions of the experimental disease, at a time when cultures from the damaged lung were negative-except the filtrable organisms described by Olitsky and Gates and by Loewe and Zeman.

Gross Pathology.-The lungs are usually voluminous and present a striking picture of acute emphysema. Although the process is often bilateral, usually one lung or parts of a lung are more extensively involved. The normal pale yellowish gray surface of the organ is mottled with deep pink in large diffuse areas. Here and there in these pink areas are small hemorrhagic foci, usually diffuse in outline.

A frothy edema fluid is often present in large quantity in the lumen of the trachea and larger bronchi. The mucous membrane of the latter regularly shows intense congestion and edematous swelling and is often stippled with minute hemorrhagic points.

On section of the lungs, no areas of firm consolidation are discoverable. The large pink areas appear to be chiefly the site of an intense congestion and edema, so that the cut surface drips a blood stained fluid. Within the confines of the pinkish lung tissue there are areas of swollen lung parenchyma of a deeper red which present the appearance of red jelly, but are compressible and moist. Often the affected portion of a lobe is a deep pink at the periphery, shading to a darker red at the hilus. In these red areas, some small dark hemorrhages, pinpoint to pinhead in size, are frequently discernable. Between the areas of involved parenchyma, the lung presents merely an intense emphysema.

Microscopic Picture.-On microscopic examination, the salient feature of the pathologic process are: (1) congestion and edema and occasional small hemorrhages in the tracheal mucosa ; (2) small hemorrhages into the walls of bronchi and the presence of red blood cells in the bronchial lumen; (3) emphysematous overdistension of alveolar air spaces; (4) congestion, and in many places, edema of areas of lung parenchyma both interstitial and interalveolar; (5) hemorrhagic extra-

15. The animals were killed by a single sharp blow over the suboccipital region. Death was instantaneous. 
vasations into the interstitial tissue of the alveolar walls with a great tendency for the hemorrhage to break into the alveolar air spaces, filling smaller or larger groups of them with red blood cells; (6) absence of ordinary cellular inflammatory exudate; (7) occlusion of numerous interalveolar and interstitial capillaries and arterioles by blood platelet thrombi. Except for the vascular lesions, all the other phenomena were also observed by Olitsky and Gates in their experimental animals.

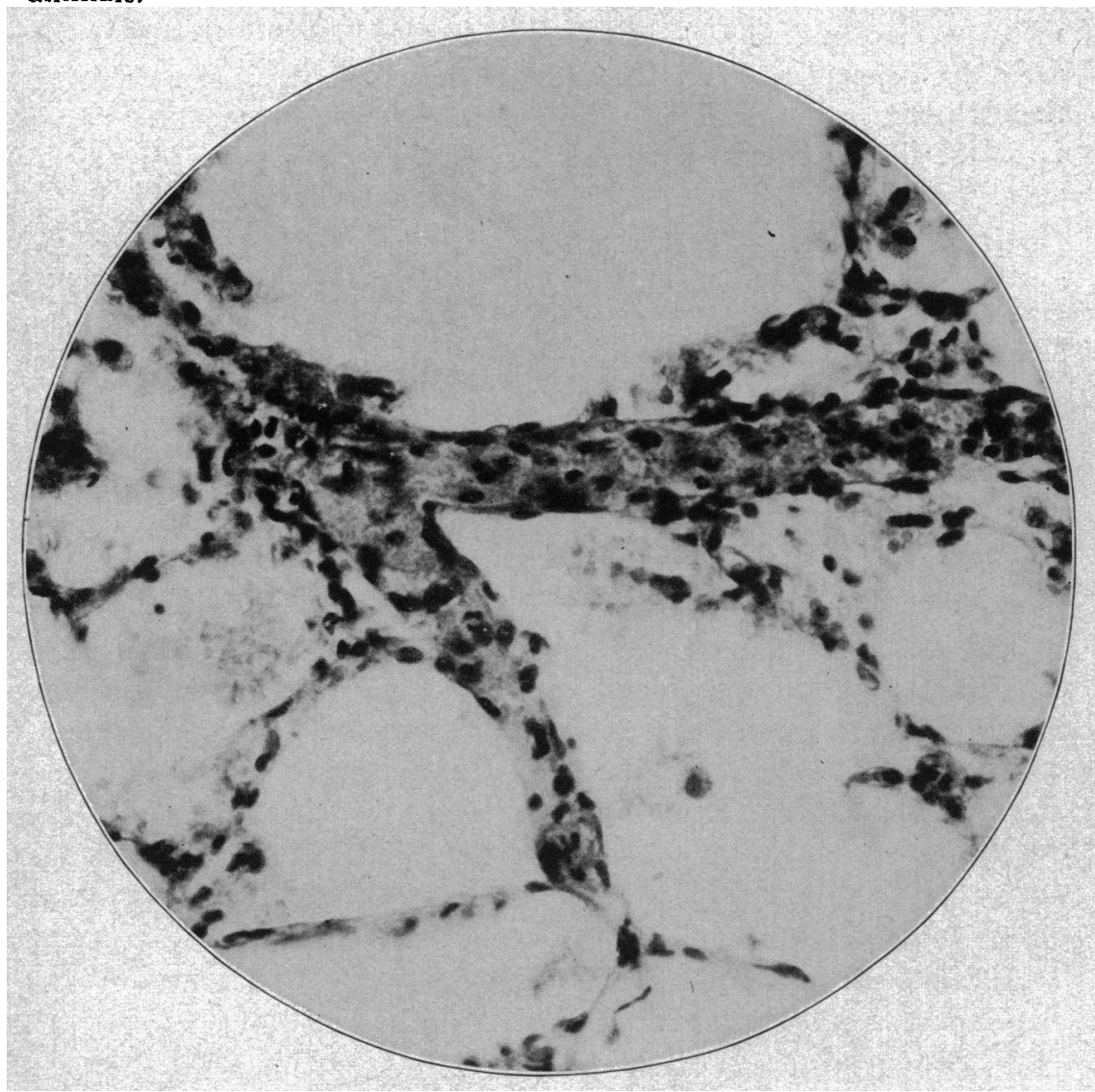

Fig. 3.-Section showing dilated and branching capillary occluded by blood platelet thrombus. The endothelial cells of the capillary wall are swollen and at several sites can be seen to have undergone proliferation. $(\times 400$.

The changes in the tracheal and bronchial mucosa occur with great regularity. Similarly, the tendency to emphysematous overdistension of the alveolar air spaces seems to be a characteristic phase of the pathologic process. It is to be seen in parts of the lung parenchyma which show as yet no evidences of edema or of hemorrhagic extravasa- 
tion. It is not caused by the mechanical presence of the injected material within the respiratory tract, for by the time the first symptoms of illness appear in the inoculated animals (six or more hours after the injection) the inoculated fluid is undoubtedly entirely absorbed. The cause is more likely to be the narrowing of the bronchial lumina due to congestion and edematous swelling of the lining mucosa. The presence of the profuse frothy mucoserous fluid within the lumen of the bronchial tree, also probably contributes to the respiratory difficulty. The development of these changes in the bronchi is coincident with the onset of asthma-like breathing and the presence of numerous moist râles on auscultation of the chest of affected animals.

Congestion of the lung parenchyma is usually patchy in distribution, as is the edema. In large and small areas, both the interalveolar tissue and the lumina of alveoli are filled with serum. Similarly, the hemorrhagic extravasations are patchy in distribution and involve the interalveolar connective tissue as well as the lumina of alveoli. These changes correspond with considerable accuracy to the lesions found in the lungs in the earliest human cases of influenza.

Blood Platelet Capillary Thrombi.-The minute intracapillary blood platelet thrombi constitute a significant and conspicuous feature of most of the experimental material that has thus far been studied. They have been found in the lungs of animals that were killed shortly after the development of the first manifestation of illness, and probably precede and are in part responsible for some of the features of the pathologic picture.

The occluded capillary loops are always widely dilated; the lining endothelium is swollen and often shows beginning proliferations; the lumen is occupied by a mass of blood platelets, scattered through which are sometimes a variable number of mononuclear and polymorphonuclear leukocytes. In the vicinity of such an obstructed capillary or minute arteriole, either edema or interstitial and intra-alveolar hemorrhages are usually present. But similar changes can sometimes be seen in capillaries within areas of lung parenchyma which show as yet merely some interstitial congestion and emphysema. The lesion has also been seen in the mucous membrane of the trachea of these animals.

Emulsions and filtrates of the lungs of animals showing such lesions have induced the experimental disease when injected into the trachea of other animals. The virus has thus been passed in series through several transmissions and in each transmission the capillary lesions have been seen. They, therefore, probably represent an essential and characteristic feature of the pathologic process in the earliest stages of experimental influenza. 
Absence of Characteristic Lesions in Control Animals.-This belief is strengthened by the fact that we have not encountered this lesion of the lung capillaries in an extensive experience with human and animal pulmonary diseases. We have repeatedly produced pulmonary infections in rabbits by the intratracheal inoculation of various common pyogenic organisms such as the Staphylococcus aureus, Streptococcus

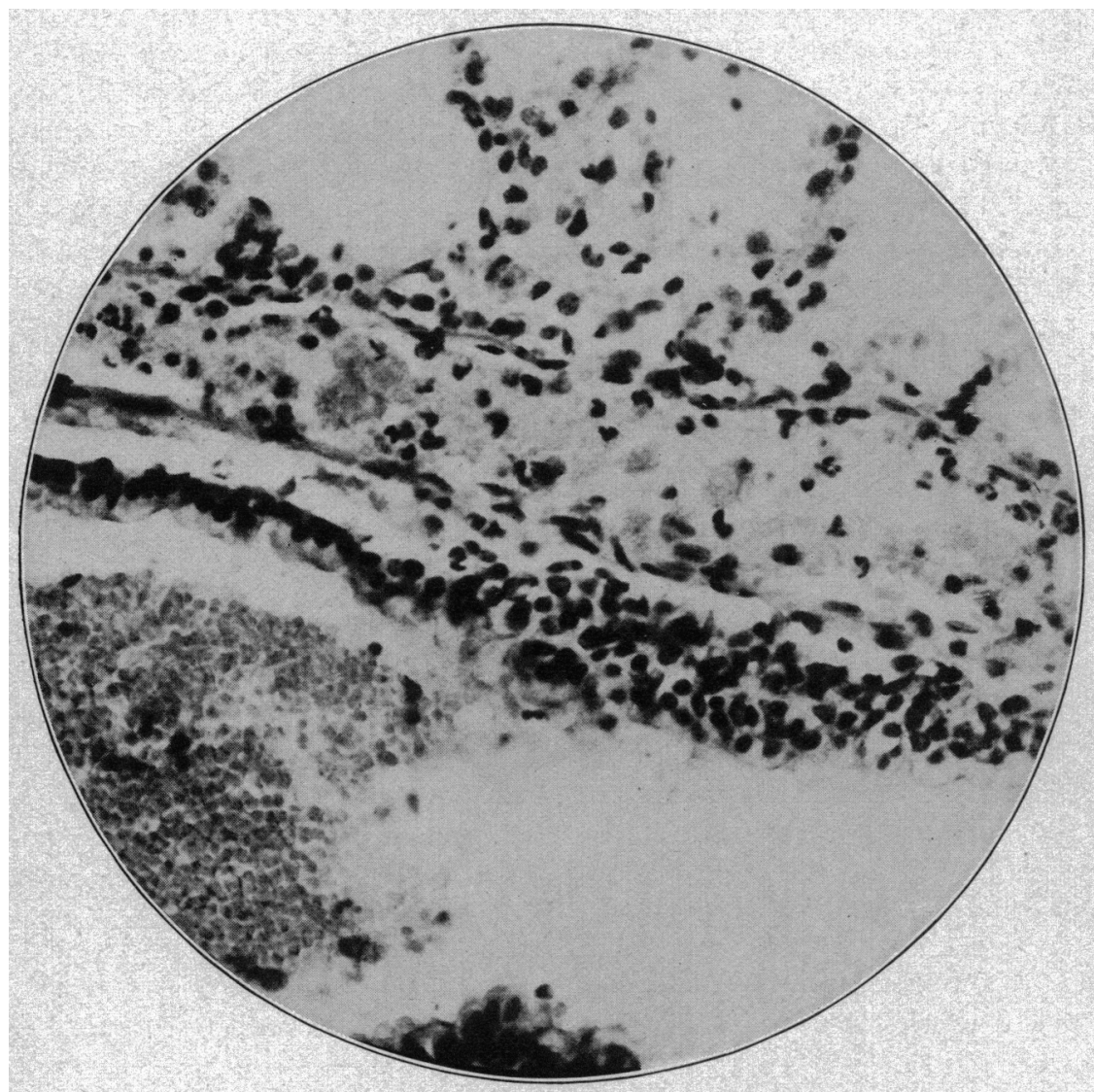

Fig. 4.-Peribronchial arteriole. The lumen of the small bronchus, which occupies the lower half of the field, is filled with red bloods cells. The adjacent arteriole which runs parallel to the long axis of the bronchus, is occluded with a blood platelet thrombus containing scattered polymorphonuclear leukocytes. The endothelium lining the arteriole, more especially on the side adjacent to the bronchioles, is very evidently in the process of proliferation. $(\times 400$.)

hemolyticus, pneumococcus, Bacillus bipolaris (distemper) and the $B$. influenzae of Pfeiffer, and have never seen anything remotely suggesting it.

Neither the capillary thrombi nor any of the other features of the experimental disease could be produced by the intratracheal injection of uninoculated culture material. In order to eliminate the possi- 
bility that the pulmonary changes in our experimental animals might be due to a purely chemical action of the injected ingredients, the control culture mediums were employed after incubation at $37 \mathrm{C}$. for variable periods of time up to fourteen months.

Significance of the Blood Platelet Capillary Thrombi-Changes similar to those seen in capillaries and arterioles in our animal lungs have also been described in the earliest stages of human influenza by Glaus and Fritzsche, Siegmund, Winternitz and Klotz. They have been searched for, though not found, by Marchand, Oberndorfer, Deitrich, Koopman and others who have also studied early examples of the disease. This may, in part, be due to the fact that in their human material the stage of secondary infection had already begun with its consequent obscuring of the primary pathologic lesions. It is more probable, however, that the greater frequency with which the capillary blood platelet thrombi occur in our experimental material is due to the fact that a much greater concentration of virus is introduced into the respiratory passages of the experimental animal than is responsible for infection in human beings.

As a result of a pathologic study of two fulminating cases of the disease, Oberndorfer was the first to voice the belief that the influenza virus produced a primary damage on the intima of small vessels. A similar viewpoint, based on a study of human material, has been expressed by Dietrich, Winternitz, Klotz, Glaus and Fritzsche, and Soerenson.

The lesions which we have been able to produce experimentally by means of influenza filtrates and influenza cultures leave little room for doubt that the virus possesses a peculiar affinity for the vascular endothelium. Numerous short stretches of capillaries and arterioles become widely dilated, apparently due to a complete loss in their normal tonus-and through their walls there occurs a great exudation of serum or a simultaneous extravasation of serum and red blood cells, which fills the interlobular and interalveolar connective tissue spaces and the alveoli in more or less sharply limited areas of vascular distribution. The blood platelet thrombi which become deposited in some of these dilated and damaged capillaries and arterioles are also to be encountered occasionally in parenchyma which is not as yet involved in any serous or hemorrhagic extravasation, so that they undoubtedly also represent an expression of the primary damage to the vascular endothelium. They are certainly not secondary to the other parenchymal changes.

Winternitz laid especial emphasis on the. frequency with which necrosis of interalveolar septa occurs, and he regarded it as one of the characteristic effects of the disease. In the experimental material, necrosis and even rupture of interalveolar septum was occasionally encountered, but at the stage of the process at which the study was 
necessarily made, this was not as yet a wide spread or conspicuous phase of the pathologic picture. In one of the fulminating cases of Glaus and Fritzsche, which probably represented a stage of the process somewhat later than our experimental disease, they call attention to the fact that though the necroses are numerous, the capillary thrombi are also to be seen outside of the necrotic areas. "In many instances," they state, "one can observe how the necrosis occurs in the immediate proximity of these thrombosed vessels." The experimental observations confirm this viewpoint that the capillary thromboses precede the necrosis and are not secondary to it.

Aplastic Character of the Inflammatory Exudate.-Perhaps the most striking feature of the microscopic picture in our experimental material and one which alone serves to differentiate it from any other type of pulmonary inflammatory process is the aplastic character of the alveolar as well as interstitial exudate. An occasional proliferated endothelial cell is encountered. But unlike any other type of pulmonary infection, no polymorphonuclear or other leukocytes are seen in the early stage of the process. The exudate consists almost wholly of serum or of serum and red cells. Although other pathologists have noted this unique feature of the process in early uncomplicated human influenza, Winternitz and his co-workers have especially emphasized the observation that "in the early stage, one of the most outstanding features is the absence of polymorphonuclear leukocytes in the reactionary process."

As soon as the secondary bacterial invasion occurs, an extensive leukocytic invasion rapidly changes this phenomenon and thereby completely obliterates the specific features of the pathologic picture. In the experimental animals such a transformation was observed to occur as early as forty-eight hours after the onset of the disease.

Yet even before this occurs, polymorphonuclear leukocytes are encountered in abnormal numbers within the blood platelet capillary thrombi. At a time when the alveolar and interstitial exudate, except for red cells, is still absolutely aplastic, many of the thrombi in occluded capillaries and arterioles are seen to be invaded by occasional proliferated endothelial cells and by polynuclear leucocytes in fair numbers. The aplastic nature of the other parenchymal changes would indicate that the virus of influenza does not possess the slightest chemotactic property. The appearance of leukocytes only within the capillary thrombi is, therefore, probably initiated by the positive chemotaxis exerted by the presence of the damaged endothelium within these vessels. In fact, their occurrence solely in the occluded vessels is evidence that damage has occurred at these sites and indicates that this damage, and not mere mechanical factors, is responsible for the blood platelet deposition. 
Winternitz, Wason and MacNamara described a hemogeneous hyalinlike necrosis of the epithelium lining the trachea and bronchi, and, mainly for this reason, they likened the pathologic picture of the disease to that produced by the poisonous gases employed during the war. We have failed to observe this superficial necrosis in our experimental material, as have most other pathologists in their early examples of human influenza. Therefore, we feel that it is probably caused by a combination of the primary damage to the mucosa and the secondary infection and that it represents a complication rather than an essential feature of the primary pathologic process.

\section{SU MIMARY}

The following pulmonary lesions were produced in rabbits by the intratracheal inoculation of Berkefeld filtrates of nasopharyngeal washings from early cases of influenza or of cultures of the filtrable punctiform bodies cultivated from such washings by Loewe and Zeman:

(1) Congestion, edema and small hemorrhages in the mucous membrane of the trachea and bronchi.

(2) Presence of a profuse, slightly blood-tinged, frothy, serous fluid in the lumen of the bronchi.

(3) Diffuse patchy distribution of red, jelly-like lesions throughout the lungs.

(4) Intense congestion of a large part of the intervening lung parenchyma and acute emphysematous overdistension of alveoli.

(5) Widespread exudation of serum and extravasation of red blood cells from the vessels, filling interstitial tissues and groups of alveolar air spaces.

(6) Aplastic character of this exudate.

(7) Aneurysmal dilatation of short stretches of capillaries and arterioles and sometimes closure of the lumen of these vessels at these sites by blood platelet thrombi.

(8) Tendency to early secondary invasion with pyogenic organisms, which then induce a rapid purulent infiltration, thereby completely obliterating the primary and specific picture of the disease.

These characteristics have all been observed in human influenza by pathologists who have had the opportunity to study the lungs of patients with fulminating disease who died within the first few days of their illness and before secondary infection had occurred. The lesions as they occurred in these early cases have been regarded as constituting a specific pathologic picture. Our experimental lesions correspond with considerable accuracy to those of human influenza and they cannot be confused with the changes produced by any other pathogenic organism either in human beings or in animals. 
The unusual nature of the pathologic process in influenza is apparently due to the fact that the virus of this disease possesses a remarkable affinity for the vascular endothelium. The virus gains access via the respiratory passages, and is rapidly absorbed through the mucous membrane of the trachea, bronchi and bronchioles. Apparently, as a result of its peculiar endotheliotoxic property, it then rapidly calls forth a profuse outpouring of serum and red blood cells in patchy areas throughout the lungs.

Further evidence of this vascular damage is to be seen in the complete loss of tonus in many capillary loops and in the early occlusion of many capillaries and minute arteroles by blood platelet thrombi. This phenomenon, though observed in the lungs of some of the earliest human cases of influenza that were studied pathologically, can be regularly produced experimentally with influenza virus and influenza cultures and is of considerable pathogenetic significance. 